\title{
Moly: a prototype handheld three-dimensional digitizer with diffraction optics
}

Douglas A. Lyon

Fairfield University, dlyon@fairfield.edu

Tom Ditto

Follow this and additional works at: https://digitalcommons.fairfield.edu/engineering-facultypubs Copyright 2000 Society of Photo-optical Instrumentation Engineers (SPIE) Available at http://dx.doi.org/10.1117/1.602337. One print or electronic copy may be made for personal use only. Systematic electronic or print reproduction and distribution, duplication of any material in this paper for a fee or for commercial purposes, or modification of the content of the paper are prohibited.

\section{Peer Reviewed}

\section{Repository Citation}

Lyon, Douglas A. and Ditto, Tom, "Moly: a prototype handheld three-dimensional digitizer with diffraction optics" (2000). Engineering Faculty Publications. 78.

https://digitalcommons.fairfield.edu/engineering-facultypubs/78

\section{Published Citation}

Ditto, Tom, and Douglas A. Lyon. "Moly: a prototype handheld three-dimensional digitizer with diffraction optics." Optical Engineering 39, no. 1 (2000): 69-78.

This item has been accepted for inclusion in DigitalCommons@Fairfield by an authorized administrator of DigitalCommons@Fairfield. It is brought to you by DigitalCommons@Fairfield with permission from the rightsholder(s) and is protected by copyright and/or related rights. You are free to use this item in any way that is permitted by the copyright and related rights legislation that applies to your use. For other uses, you need to obtain permission from the rights-holder(s) directly, unless additional rights are indicated by a Creative Commons license in the record and/or on the work itself. For more information, please contact digitalcommons@fairfield.edu. 


\section{Moly: a prototype handheld three-dimensional digitizer with diffraction optics}

\author{
Tom Ditto \\ DeWitt Brothers Tool Company, Inc. \\ P.O. Box 4000 \\ Ancramdale, New York 12503 \\ Douglas A. Lyon \\ University of Fairfield \\ Computer Engineering Department \\ Fairfield, Connecticut 06430
}

\begin{abstract}
A working handheld 3-D diffraction range finder, nicknamed Moly, is demonstrated. This prototype is distinguished by a far-field magnification feature that is made possible by use of chirped frequency diffraction grating optics that reverse the perspective foreshortening typical of conventional triangulation range finders. This new type of 3-D profilometer illuminates its target with a collimated laser projector that produces a rectangle-shaped sheet of light of uniform width at all working distances. Moly also employs dual magnetic wave detectors to facilitate freedom of movement for both the digitizing instrument and the subject. The instrument was designed primarily to digitize human faces and figures for applications in art and medicine. () 2000 Society of Photo-Optical Instrumentation Engineers. [S0091-3286(00)00801-1]
\end{abstract}

Subject terms: diffraction, range finder; holography; grating; three-dimensional; profilometry, machine vision; metrology.

Paper SM-08 received June 29, 1999, revised manuscript received Aug. 10, 1999, accepted for publication Aug. 10, 1999

\section{Background}

We trace our experimentation with 3-D digitizers back to our Pantomation ${ }^{1}$ computer interface circa 1983, a spatial tracking system that used video camera transducers and a modified light pen computer interface architecture to follow dancers' movement in real time. In 1983, Pantomation was used in conjunction with a theatrical laser projector to scan 3-D surfaces. This early experimentation followed the strategy of triangulation and suffered from the limitations including perspective foreshortening, occlusion liability, and fixed field of view that are endemic to the triangulation method.

The weak points of triangulation motivated the discovery of a new method of range finding based on diffraction that offered some potential improvements on these limitations. ${ }^{2}$ A 1987 prototype diffraction range finding system demonstrated a synchronized scanning feature that can overcome the fixed-field-of-view problem. ${ }^{3}$ By synchronized scanning, we mean that the camera and the projected laser beam were functionally coaxial wherever scanned. The synchronized scanner prototype employed a type of surface relief transmission plane grating that is commercially available as an inexpensive embossed plastic material. The 1987 prototype also enjoyed redundant target views that reduced occlusion liability when compared to other published methods of synchronous scanning. However, perspective foreshortening remained a problem.

To overcome the perspective foreshortening and to further reduce occlusion liability, we invented a diffraction optical element based on chirp gratings. Unlike the plane gratings used in our prior demonstrations, the chirp grating has a variable pitch. The change in pitch results in a magnification feature that can be tailored to target distance.

\section{Theory of Operation}

The diffraction range finding method works on the principle that higher order diffraction images are displaced in position (relative to their central zero order) as a function of target distance. For this method to work, targets must radiate energy in periodic waves, although neither monochromatic nor coherent waves are mandatory. Targets can be self-illuminated, or they can be designated by an interrogating beam, typically a laser so as to take advantage of a laser's superior depth of focus. The energy radiated from the target is received through a diffraction grating. In the light frequency region of electromagnetic radiation, the receiver can be a video camera, but the diffraction range finding method is extensible to any form of energy transmitted by periodic waves. In the visible light regime, a lens is placed between the grating and the transducer. The lens forms a perspective center through which rays can be traced in a geometric analysis.

Specification of a diffraction range finder that will produce a desired higher-order image displacement for a given target distance can be made using equations that are derived from the well-known grating equation:

$\sin (r)+\sin (i)=n \frac{\lambda}{p}$,

where

$r=$ angle at which diffraction images are reconstructed

$i=$ angle of incidence of a wavefront

$n=$ diffraction order, an integer

$\lambda=$ wavelength of incident energy 


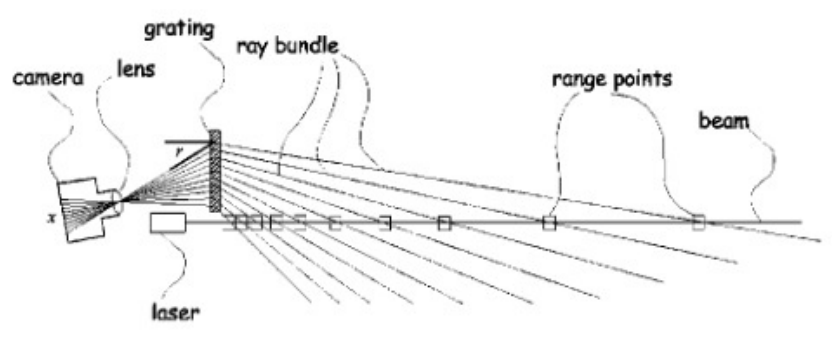

Fig. 1 Diffraction range finder using a plane grating. In the diagram, rays are placed in equal steps along a camera focal plane. Note that corresponding range points are not in equal steps but increase geometrically in spacing as a function of range.

$p=$ pitch of the grating.

In the near-field where the wavefront striking the grating is appreciably spherical, angles $i$ and $r$ are dependent on the distance from the grating of a target point source of energy. The change of slope in the spherical wave as a function of target proximity to the grating causes the change in higher order image position. A measurement of the angle $r$ at a receiver at distance $d$ from the grating will yield the range $D$ of a target according to the relationship

$D=d \tan (r) \frac{\left\{1-[n(\lambda / p)-\sin (r)]^{2}\right\}^{1 / 2}}{n(\lambda / p)-\sin (r)}$

In the special case of visible radiation, the receiving angle $r$ is typically acquired using a camera with a lens of focal length $F$ in conjunction with a focal plane where a displacement $x$ along one axis of the focal plane can be measured. In this setting it can be said that

$r=\arctan \left(\frac{x}{F}\right)$

A range camera devised according to this principle using a plane grating is diagrammed in Fig. 1. Since we have parameters in Eqs. (2) and (3) that can be known, measurements of displacement $x$ of a higher-order image formed at the image plane can be correlated to a range distance $D$. However, for any equal displacement $x$ distances $D$ increase geometrically as a function of range. This effect is called perspective foreshortening, a hyperbolic dependency of displacement of higher order diffraction images as a function of target range.

Triangulation and stereoscopy range-finding methods also produce a loss of resolution in proportion to the square target distance, because the view at the receiver depends on image formation through a perspective center, the camera lens. 4

The problem of perspective foreshortening in triangulation range finders is further aggravated when the Scheimpfug condition ${ }^{5}$ is used to better maintain focus over the entire range finder depth of field. Without giving rigorous proof here, we use the two diagrams in Figs. 2(a) and 2(b) to illustrate how the conventional methods of perspective correction achieved in a photographic camera by means of
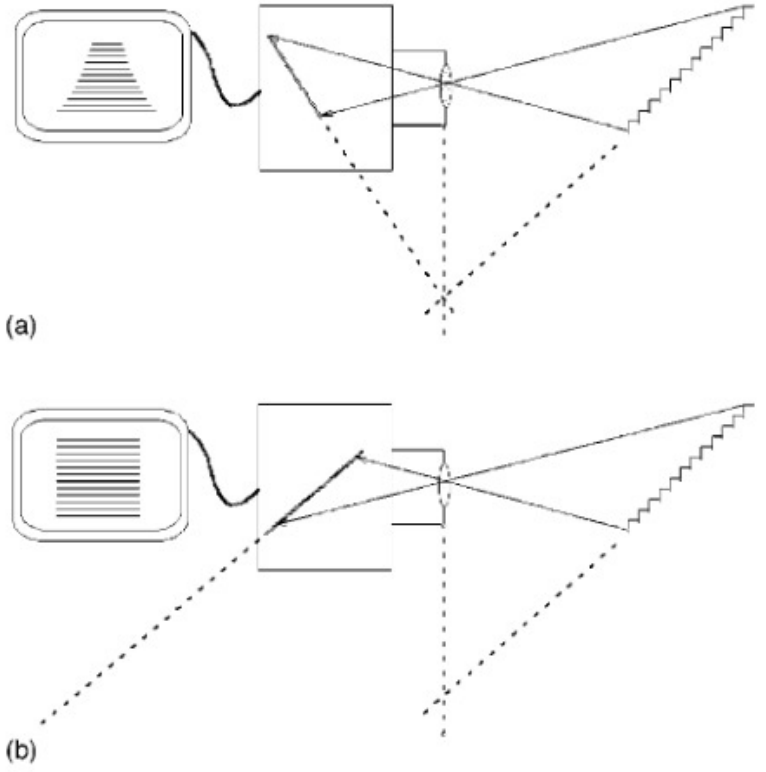

Fig. 2 (a) Scheimpflug condition compensates for depth of focus in range finders by tilting the focal plane according to a geometric rule. The focal plane, the lens plane, and the target plane all intersect at a common point. This method of focus correction aggravates perspective foreshortening. (b) Perspective correction is accomplished by a reciprocal geometry to the Scheimpflug condition whereby the focal plane and the range plane are approximately parallel.

focal plane rotation is contradicted when the Scheimpflug condition is invoked to compensate for focus variation from the near field to the far field.

It is possible to correct perspective foreshortening in a diffraction range finder by changing the pitch of the grating along its length. We can express the angle of incidence of a ray on the diffraction grating as

$i=\arctan \left(\frac{x d}{F D}\right)$.

Substituting for $i$ and $r$, as per Eqs. (3) and (4), we can solve for $p$ in the grating equation [Eq. (1)]:

$p=\frac{n \lambda F D}{2 x d}\left[1+\left(\frac{x d}{F D}\right)^{2}\right]^{1 / 2}$.

Using this equation, specifying an arbitrary displacement $x$ on the focal plane of a camera and an arbitrary distance $D$ of a target a grating pitch will determine an appropriate local grating pitch $p$. We can thus model gratings that would show a linear displacement of diffraction images as a function of even steps of range distances. The resultant grating models are variable pitch gratings with a hyperbolic frequency chirp.

To graph this relationship, we calculate an abscissa in terms of position along the grating length $L$, where

$L=\frac{x d}{F}$. 


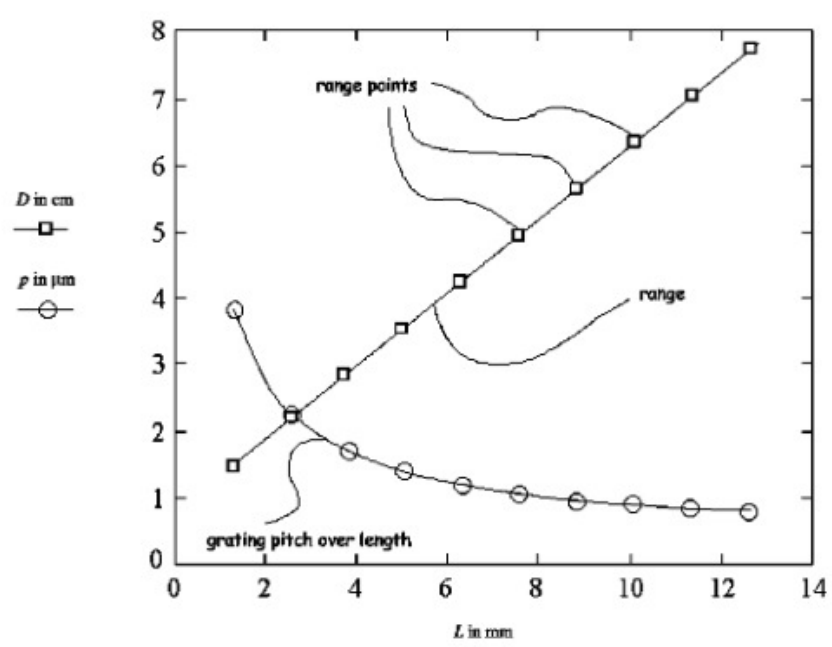

Fig. 3 Typical graph (not Moly) of range $D$ versus the position $L$ along a grating compared with the corresponding pitch $p$ of the grating at position $L$.

Figure 3 shows a typical specific calculation that is applied to the camera model shown in Fig. 4. In this model, as range distance changes, the displacement along the focal plane of the camera changes in a linear relationship to range. For this to work, the pitch (i.e., wavelength) of the grating must decrease as the distance increases. The rate of change in the pitch is itself hyperbolic and not linear.

A more general model for a diffraction range finder model allows for rotation of the grating plane relative to the receiver, as per Fig. 5. An equation that can be used reads:

$D=\frac{\Gamma d \tan \gamma}{\cos \alpha-\Gamma \sin \alpha}$

where

$\gamma=\rho+\arctan (x / F), \beta_{n}=n \lambda / p$ and

$\Gamma=\beta_{n}^{-1}\left[1-\left(\beta_{n}-\sin \gamma\right)^{2}\right]^{1 / 2}-\sin \gamma$.

With the grating inclined, the number of grooves used increases, the resolution increases as per the Raleigh criterion, and also the Bragg angle is more easily achieved. A parameter in Eq. (7) for this rotation, which we call $\rho$, must be included in our calculations, and the angle of the illumination relative to the grating plane is now accounted for by variable $\alpha$. As a critical measurement of performance, we have specified the occlusion immunity angle $\beta$ and derived a trigonometric function to predict it:

$\beta=\left[\arctan \left(n \frac{\lambda}{p}\right)\right]-\sin (r)-\alpha$.

\section{Motivation for Moly}

We previously built a prototype synchronous 3-D scanner using a plane grating, but the newly invented chirp grating range finder did not afford us the same synchronous scanning option. To provide area scanning built from profiles, we contemplated a handheld unit that assumed interactive scanning by the instrument operator. Devices of this type

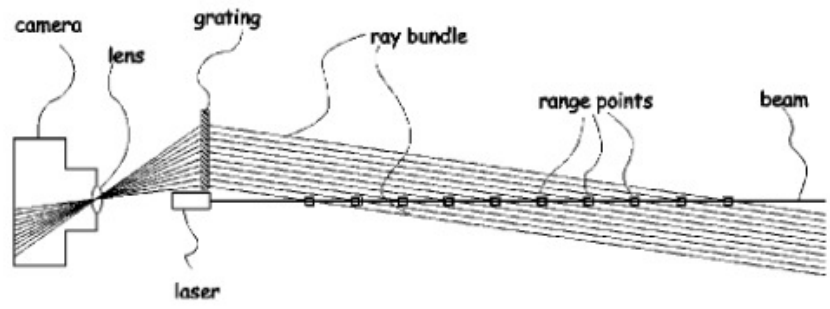

Fig. 4 Diffraction range finder using a chirp grating designed to linearize the relationship between range distance and displacement of the higher order diffraction image along the focal plane of a camera The box tick marks correspond to the graph in Fig. 3.

must have a localizer, that is, a reference point on the scanner that is tracked relative to the coordinate space of the subject being scanned. From the available options for localization we chose magnetic wave detection, a technology that was compatible with our intended subjects, human faces and figures.

Magnetic wave detectors enjoy occlusion immunity that is superior to line-of-sight methods based on triangulation, but the magnetic field is subject to distortion by conductive metals. As a result, our machinist, Paul Friedlander, chose a plastic material, molybdinum-di-sulfide impregnated nylon, from which he coined a name for our prototype, "Moly."

We know from the history of 19th-century portrait photography that special problems arise when sitters were required to remain motionless during the long exposures dictated by the insensitive early photographic recording materials and slow camera lenses. As a handheld profilometer we foresaw a similar problem in Moly, since it would be acquiring a single $200-\mathrm{mm}$-wide line profile each $33 \mathrm{~ms}$ and would therefore require many seconds to fully scan a face or other body feature. To allow the subject some freedom of movement, we developed a dual-localizer system, one for the scanner and another that could be fixed on the subject. With this adaptation, the subject would generate a coordinate space relative to Moly. Indeed, even with Moly stationary, a subject could scan himself through his own movement. While we were working on our dual tracker, we learned of a similar system for a triangulation-based profilometer that is now marketed by Polhemus, ${ }^{6}$ the manufacturer of our localizer.

We used the metaphor of a "3-D paintbrush" to guide our design. The concept is illustrated in Fig. 6. The bristles of our paintbrush were virtual. They sensed a profile taken along a stripe of illumination projecting from the scanner. The metaphor introduces a projector line geometry not found in typical triangulation sensors. We learned from users of triangulation systems that the fan-shaped field of illumination typical of their laser projectors was difficult for users to judge, because in the near field the acquisition stripes were smaller than longer acquisition stripes in the far field. Our projection system specification of either a collimating lens or hologram was going to be more complex to build but offered the user a functional improvement when compared to typical triangulation profilometers, because the sheet of light was rectangular in shape.

Our design required consideration of occlusion liability, the angle inscribed between a projected laser line and a corresponding sight line at the receiver. In a triangulation 


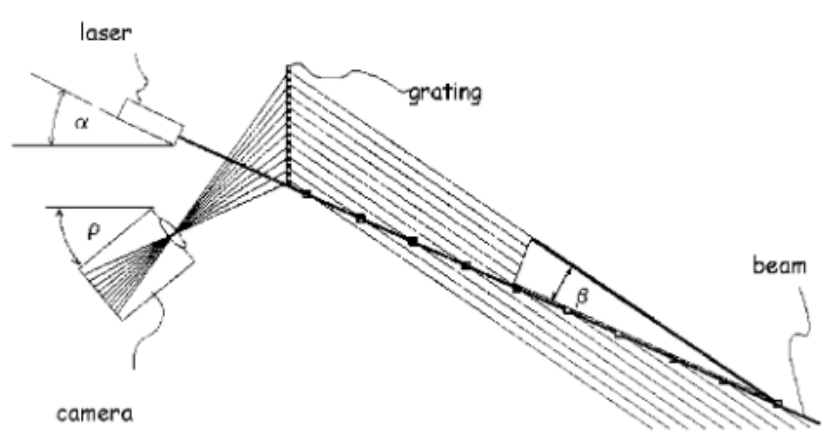

Fig. 5 Occlusion liability angle $\beta$ is the angle inscribed between the view ray and the illumination beam. The rotation of the focal plane relative to the grating is designated as $\rho$, and the illumination beam is rotated relative to the grating plane normal by angle $\alpha$.

system the extreme case would be a 90-deg separation, in which case the camera sees a 1:1 profile, that is, a sideways view. However, such a radical angle is impractical, calling for the camera to be virtually embedded inside its target in most instances. In commercially available triangulation systems, the occlusion liability angles are rarely greater than 45 deg. We designated a 28-deg occlusion liability angle, which appeared on survey to be an industry convention. However, unlike triangulation systems, the occlusion liability angle in Moly would be constant, because the sight lines to the receiver would be parallel.

Of course, we could not proceed without a diffraction grating made to our specification. Unlike plane gratings, chirp gratings are not commonplace in spectroscopy and cannot be bought off the shelf. Yet we knew that every hologram is itself a diffraction grating, so we turned to holographers to make our grating. Our ignorance of the process proved to be one of the most interesting turns in the project, because we took it on advisement from our first subcontractor that a desired hyperbolic chirp was not an option if the grating was made holographically. We learned later that, in fact, a hyperbolic chirp is the only option in a simple holographic grating made using the Leith-Upatnieks ${ }^{7}$ method, but in our confusion we developed design tools that were premised on the notion that the chirp rate would be linear. We also came to respect the problems of grating efficiency. Our first proposed design looked almost exactly like the paintbrush in Fig. 6, but the diffraction angles were so shallow that the gratings proved

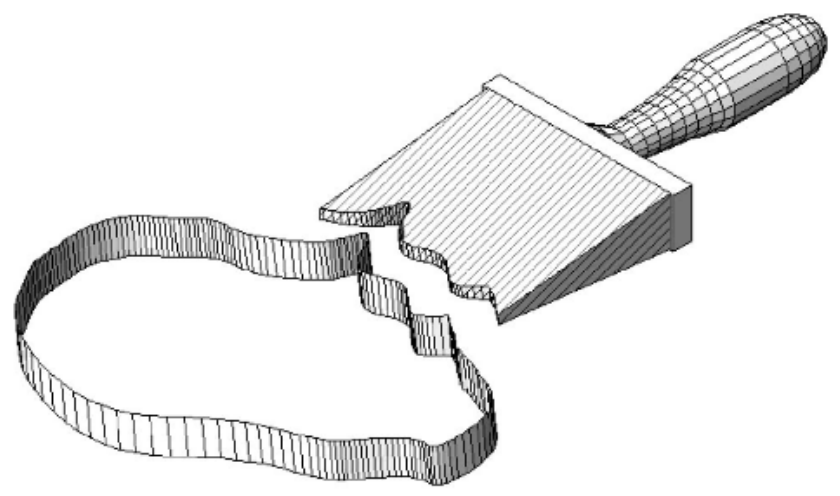

Fig. 6 The 3-D paintbrush concept. extremely inefficient. As our expertise grew, we learned that the safest way to design an efficient grating was to have the diffraction occur near the Bragg angle, and this informed the design of the holographic grating made for Moly.

\section{Design Tools}

We developed two routes to design our optical system, classical trigonometry and interactive computer software that uses numerical methods. We felt that having corroboration between the two types of model would give us better confidence when we committed to a grating specification.

The geometric optics of diffraction range finding are discussed in Sec. 2, but the devil is in the details. For holographic materials, exposures are simplest with $\rho$ near to 45 deg. In Moly, $\rho$ is $41 \mathrm{deg}$. Furthermore, in the predictions that constitute Figs. 7 and 8 , we have assumed a $1 / 2$-in. CCD with a $16-\mathrm{mm}$-focal-length lens. The target is illuminated by a laser diode with a wavelength of $636 \mathrm{~nm}$ that is rotated relative to the grating plane by $22 \mathrm{deg}$, the angle we call $\alpha$. Our grating calculation has a linear chirp from 450 to $550 \mathrm{~nm}$ over its length $L$ of $12.7 \mathrm{~cm}$, the long side of the $4 \times 5$-in. holographic plate to be used. The computer program MathCad6 was used to graph the calculations. Two results are shown in Fig. 7.

DiffCAD is a program written in JAVA by Lyon and takes its name from the exercise of writing a diffraction computer-assisted design program. Later expanded to demonstrate JAVA coding for many signal processing algorithms, DiffCAD is distributed in a JAVA programming textbook $^{8}$ and as shareware. ${ }^{9}$ The user can specify the pitches of a grating at the extrema, and the program calculates the grating pitches across the surface as a linear chirp. A camera can be specified with one dimension of the sensor array and the lens focal length. The angle of view of the camera can be input or can be set automatically by the program as the user drags a camera icon with a mouse. The work area has a user controllable grid for dimensioning, but many readings are returned in dialog boxes, including graphs.

DiffCAD corroborated the predictions of the geometric optics equations developed in the MathCAD and Maple symbolic environments. Figure 8 shows the original plan for Moly as rendered on the DiffCAD screen.

\section{Actual Prototype}

Our optical design tools did not account for the mechanical design of the instrument. Here we turned to a professional machinist, Friedlander, ${ }^{10}$ who is well versed in prototype assembly. This resulted in a pencil-on-paper plan that was realized in his machine shop. The design has now been entered into a 3-D CAD program, which produced the rendering in Fig. 9. The actual prototype is shown in Fig. 10.

Moly's camera and its lens were separately mounted on rails to enable adjustments, especially the rotation of the lens relative to the sensor focal plane. This adjustment, the Scheimpflug angle, is critical to depth of focus in diffraction as well as triangulation range finders. We learned empirically that a 5-deg rotation was effective. To create a collimated projected laser beam, we used a laser with an integrated line projector and a positive cylindrical lens with a focal length of $473 \mathrm{~mm}$ matched to the 20-deg fan of the 

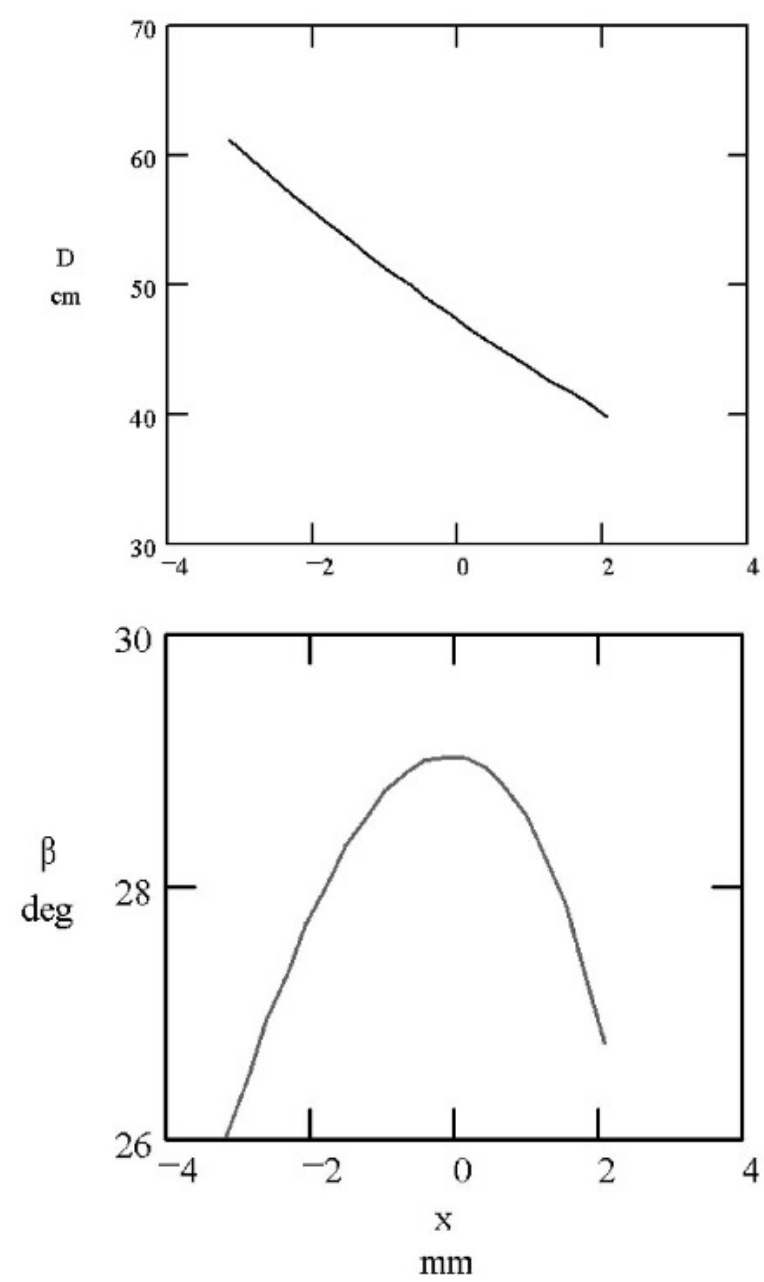

Fig. 7 Predictions for Moly's performance for range and occlusion immunity. The stand-off of $400 \mathrm{~mm}$ results from assumptions in the position of the laser. In fact, stand-off with Moly's chassis is $100 \mathrm{~mm}$. The slight variation in range linearity and occlusion liability is an artifact of the approximation that the grating has a linear chirp.

line projector. The resultant projection is a rectilinear sheet of light that matches the rectangular field of view from the grating.

The proof of the pudding is in the eating. Moly ended up being larger and heavier than we would have hoped. However, while not a paint brush, the chassis is similar to a vintage camcorder, a handheld unit from our recent memory. At $3 \mathrm{~kg}$, the unit is not steady in one hand but is easily stabilized when both hands are used. The size is traceable to the 470-mm stand-off between the laser and the cylindrical lens. This is matched by a nearly equal stand-off from the camera to the folding mirror. The weight is due, in part, to the grade of plastic used for the walls. Even cut back with open sides, as illustrated in Fig. 10, molybdinum impregnated nylon is a very dense material. The grating itself does not add significantly to the weight. Holograms are essentially flat and their only mass is their substrate. In this case it is a $4 \times 5$-in. photographic plate. We chose a lipstick style CCD for its small size. Diode lasers are now ubiquitous and of stub pencil size. Miniaturization of Moly's dimensions appears to be realizable in future iterations.
Our predictions did not include a model for grating efficiency other than to assure that the diffraction images would form near their Bragg angles. Indeed, holography is as much an art as a science, and of the three silver halide holograms made for Moly, one was far more efficient than the other two. However, all the gratings formed their diffraction images at $\pm 5 \mathrm{deg}$ of the Bragg angle, as indicated in Fig. 11. We do not have efficiency data, but Moly meets the test of performing within class IIa laser specifications with a peak intensity of $0.5 \mathrm{~mW}$ within any $7-\mathrm{mm}$ aperture along its projected stripe.

We also learned that the linear chirp model was incorrect, although it is a close approximation over the pitch spread we used. On the basis of our linear chirp model, we had expected a small amount of far-field magnification, and we wished to use this as an argument for the diffraction range finding method. Triangulation systems suffer from loss of resolution caused by perspective foreshortening, and our perspective "farshortening"' breaks with prior art in optical range finders. As it happens, we were able to introduce this effect in a subtle manner by moving Moly's camera back slightly from its originally specified location.

The lens we use is unusual. Advertised as a "pinhole", lens, ${ }^{11}$ it actually is a conversion of a zoom microscope designed in such a manner that the ray bundle inverts in front of the first element. As a result, we can empirically determine the perspective center outside the lens, a blessing for prototyping. Moreover, it appears that the narrow pinhole serves to limit an astigmatism endemic to chirp gratings in our range finding configuration. When we substitute a lens of equivalent $f$-stop and focal length, there is considerable blurring.

Our sensor is an American video standard 1/2-in. monochrome CCD camera rated at 570 horizontal lines of resolution. The "lipstick" package used puts the video processing in an outboard module connected by an umbilical. Figure 12(a) shows the video image captured in a $640 \times 480$ digitizer of a test bed made with a $50-\mathrm{mm}$-wide target in $10-\mathrm{mm}$ steps over a $180-\mathrm{mm}$ range. For measurement, we superimpose 30 pixel grid lines in Fig. 12(b).

Moly suffers from some sources of noise and geometric error. There are speckle artifacts from laser illumination. Speckle is one drawback in employing a structured illumination source based on coherent radiation. Unique to our holographic grating is the magnification both in the horizontal (the dimension from which range information is extracted) and the vertical. Note in Fig. 12 the slight increase in displacement as range increases. Some of this pseudoscopia was deliberate, that is, the slight magnification with range distance was sought to demonstrate far-field resolution. However, the vertical magnification was unexpected. It is attributed to a keystone effect. The camera is viewing the grating at such an angle that the far-field image is formed by a smaller section of the grating than the near field. Finally, Fig. 12 shows that there is a noticeable rotation in the target that is caused by a slight error in the angle of Moly's folding mirror relative to the grating plane.

The interface between Moly and the computer is a special peripheral card called SURFA by its manufacturer. ${ }^{12}$ The card is not a typical framestore but rather functions by blocking all data at the input that is not above a user-set brightness threshold and thereafter storing the passed data 


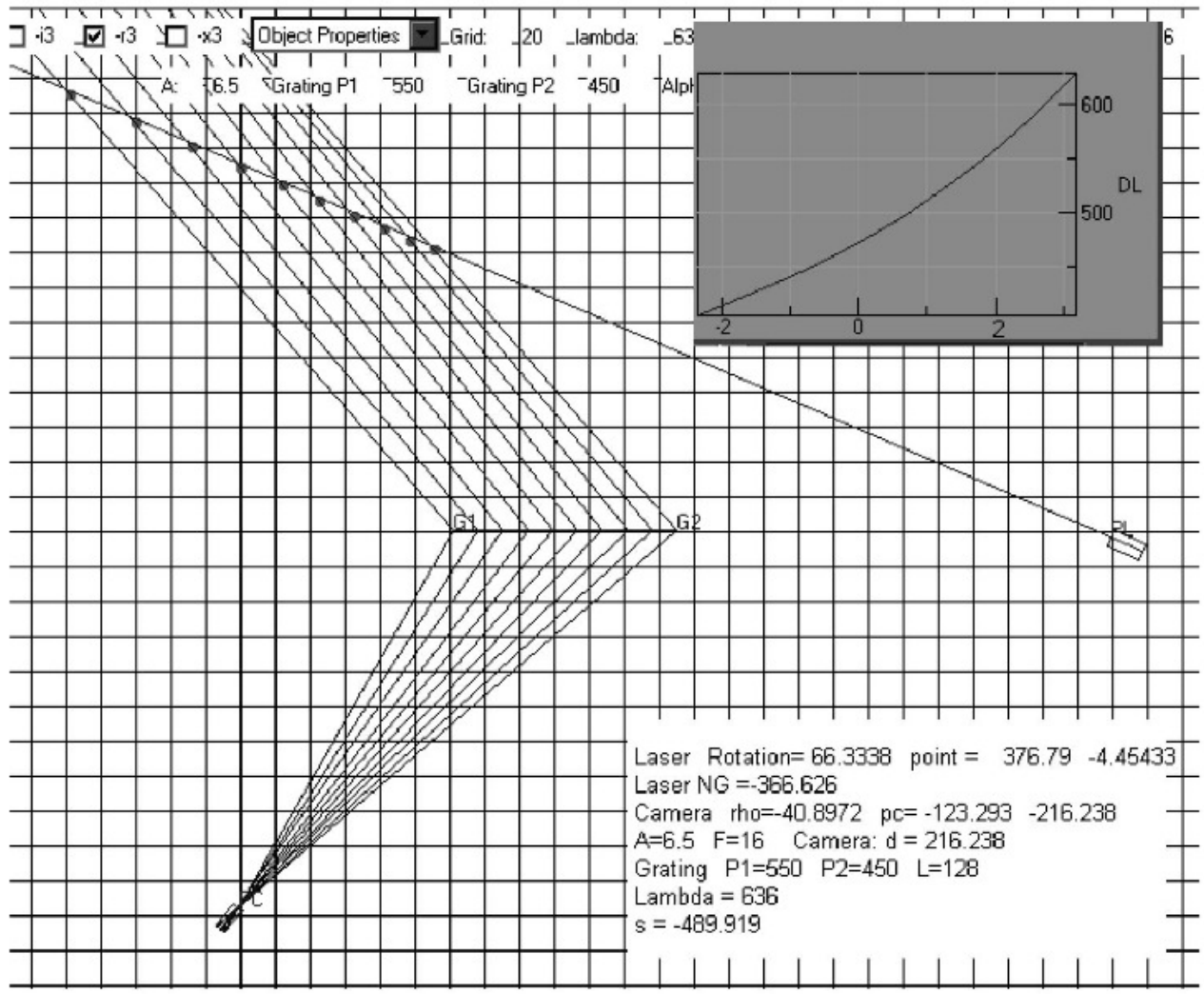

Fig. 8 DiffCAD model of Moly. The laser $P$ intersects the sight lines from the gratings $G 1$ to $G 2$ at range points, and these are used to graph the range distances as recorded on a camera sensor PC. The grid is on 20-min centers. Graph units are in millimeters.

stream in a first in first out (FIFO) pipeline for processing by an onboard digital signal processor (DSP). The card samples data on every other video field and downloads the resultant data on an ISA type PC bus where it is handled by a Windows NT program called ModelMaker published by the same manufacturer, 3D Scanners Ltd. A custom algorithm for intrapixel estimation produces range data in the DSP on the SURFA card itself. Calibration software in ModelMaker characterizes the range finder's performance. Moly achieved a root mean square (rms) error of from 0.1 to $0.2 \mathrm{~mm}$ over a 100 - to $300-\mathrm{mm}$ range, as detailed in Table 1. The rms error is based on multiple scans and

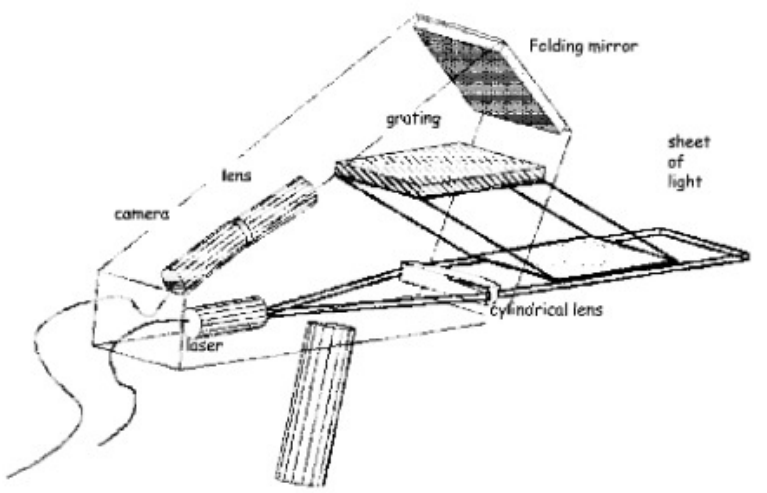

Fig. 9 Moly schematic showing the folding mirror that enables the camera to be next to the laser line projector. The sight line from the grating intersects the laser sheet of light. shows the repeatability of the scanner system, including the error introduced by the calibration system. This test was conducted using a $60-\mathrm{mm}$-wide target block, which was moved along a lathe bed that was calibrated to 0.01 in. Some of the rms error may have been introduced by the lathe. We infer from the range error measurement that any one individual scan line showed a $10-$ to $30-\mu \mathrm{m}$ optical resolution.

In any event, the accuracy of Moly's optics appear to be well within the resolution limit set by its magnetic wave

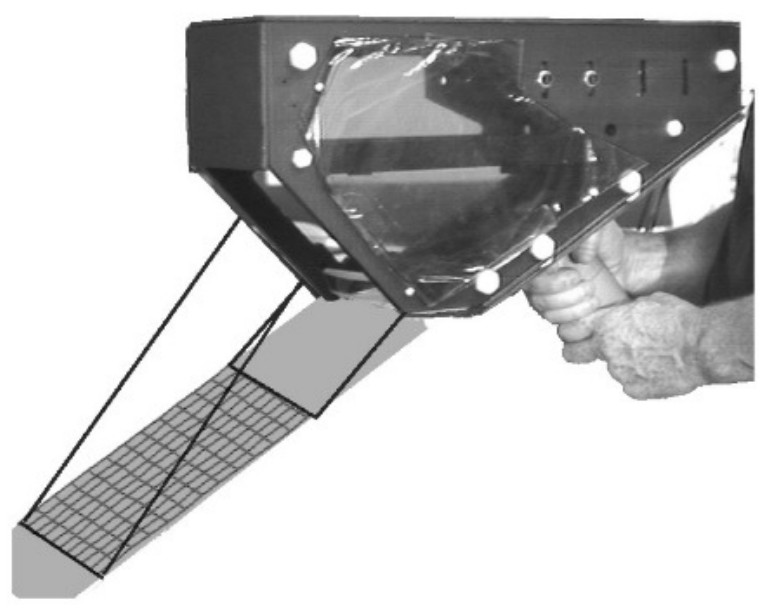

Fig. 10 Prototype of Moly. The sheet of light and the intersection with the sight line from the grating were drawn in 


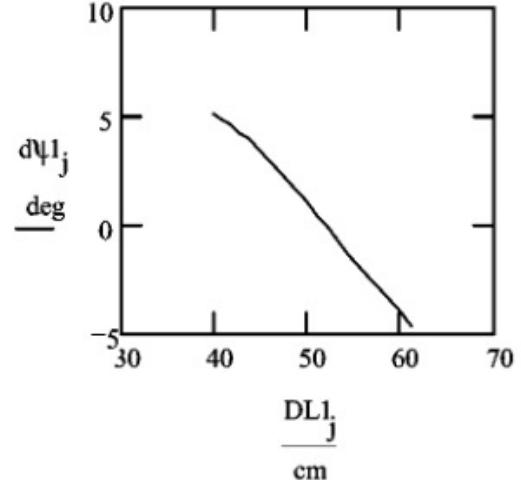

Fig. 11 Deviation from the Bragg angle $d \psi$ as a function of range distance.

localizer, which is rated at $1-\mathrm{mm}$ accuracy over a meter hemisphere. Localizer error, therefore, is the primary dimensional distortion in Moly's real-world scans. We tested Moly with a mannequin head "Biff," illustrated in Fig. 13. A data cloud made by scanning a live subject, our holographer, Rudie Berkhout, appears in Fig. 14. We look forward to perfecting the art.

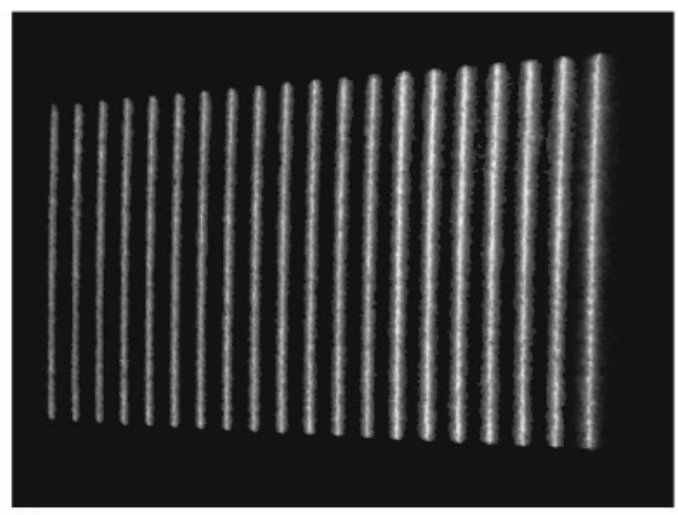

(a)

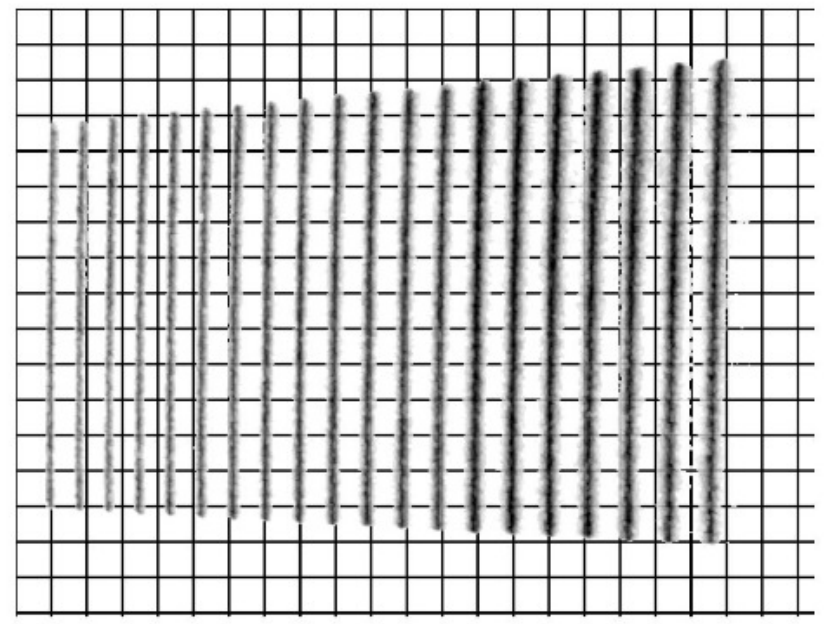

(b)

Fig. 12 (a) Calibration scan, near-field on left hand side. The target moved in $10 \mathrm{~mm}$ steps. Stand-off to Moly's chassis was $100 \mathrm{~mm}$. (b) Negative of (a) with a superimposed grid on 30 pixel centers.
Table 1 Calibration table produced by the ModelMaker program. All measurements are in millimeters.

\begin{tabular}{llllll}
\hline \hline \multicolumn{2}{c}{$\begin{array}{c}\text { Camera } \\
\text { Coordinates }\end{array}$} & $\begin{array}{c}\text { Mean } \\
\text { Width }\end{array}$ & $\begin{array}{c}\text { Mean } \\
Z\end{array}$ & $\begin{array}{c}\text { Range } \\
\text { Error }\end{array}$ & $\begin{array}{l}\text { rms } \\
\text { Error }\end{array}$ \\
\hline 27 & 230 & 59.517 & -0.288 & 0.009 & 0.19 \\
32 & 232 & 60.007 & 20.25 & 0.008 & 0.312 \\
37 & 233 & 60.13 & 40.925 & 0.025 & 0.182 \\
42 & 234 & 60.124 & 60.997 & 0.007 & 0.086 \\
47 & 235 & 60.06 & 81.262 & 0.012 & 0.09 \\
51 & 236 & 60.347 & 101.585 & 0.014 & 0.106 \\
56 & 237 & 60.186 & 121.911 & 0.006 & 0.092 \\
60 & 238 & 60.32 & 142.178 & 0.07 & 0.244 \\
65 & 239 & 59.762 & 162.631 & 0.032 & 0.211 \\
69 & 239 & 59.851 & 182.578 & 0.049 & 0.221 \\
72 & 239 & 59.869 & 203.087 & 0.019 & 0.18 \\
\hline \hline
\end{tabular}

\section{Lessons Learned and Suggestions for Future Works}

Our principal interest being diffraction range finding, the most pressing lesson learned from building Moly was to correct the far-field vertical magnification effect created by Moly's camera angle. Note that in Fig. 9, a schematic of Moly, the rays from the camera to the grating are not traced. Indeed, we failed to take this ray path into account. Had we done so, it would have revealed the keystone effect caused by viewing the grating from off axis. The question we now faced was whether moving away from the Bragg angle would result in an unacceptable loss of grating efficiency. We commissioned test gratings from holographic artist, Berkhout, ${ }^{13}$ with the specification that principal ray from the camera lens be perpendicular to the grating plane [Fig. 15(a)]. To produce this grating, he intersected a plane wave from off axis with a spherical wave perpendicular to the grating plane. The holograms he produced proved as efficient as Moly's, particularly those made where the plane wave was inclined at $60 \mathrm{deg}$ off the normal to the grating plane. However, these gratings were efficient only when rotated $11 \mathrm{deg}$ off the exposure plane [Fig. 15(b)].

Another area of investigation is the potential for a 1:1 optical reproduction of range depth. Note in Fig. 15(a) how the laser projection beam runs parallel to the grating plane. Although it is intersecting the ray bundle at a nominal 30deg occlusion liability angle, the camera will record depth information as if it were looking at the target from $90 \mathrm{deg}$.

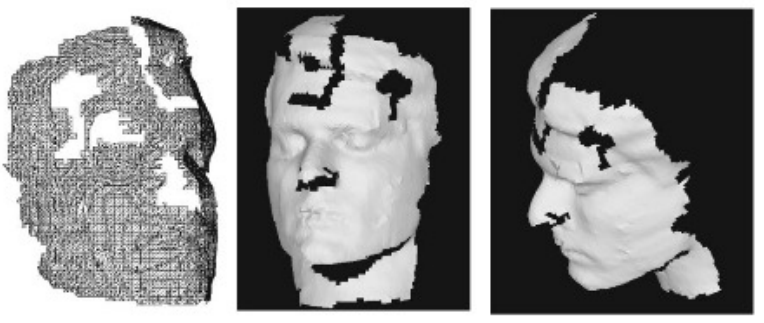

Fig. 13 First scan of a head made with Moly. Occlusion artifacts and drop outs notwithstanding, we recognized the face. 


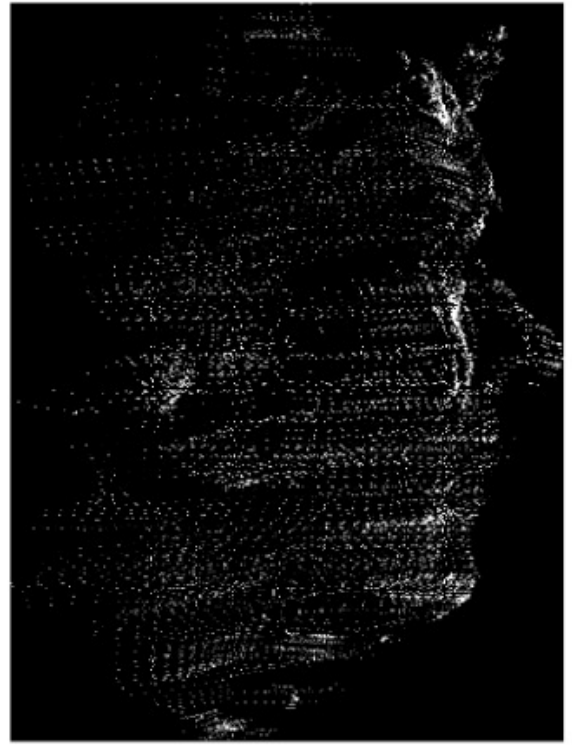

Fig. 14 Raw data cloud of a live subject using the dual tracker that allowed free head movement.

We used a Berkhout grating in a trial. Our target was a step block. Figure 16 shows the block as photographed from the side with the acquired diffraction range image superimposed, and they are a good match. The occlusion

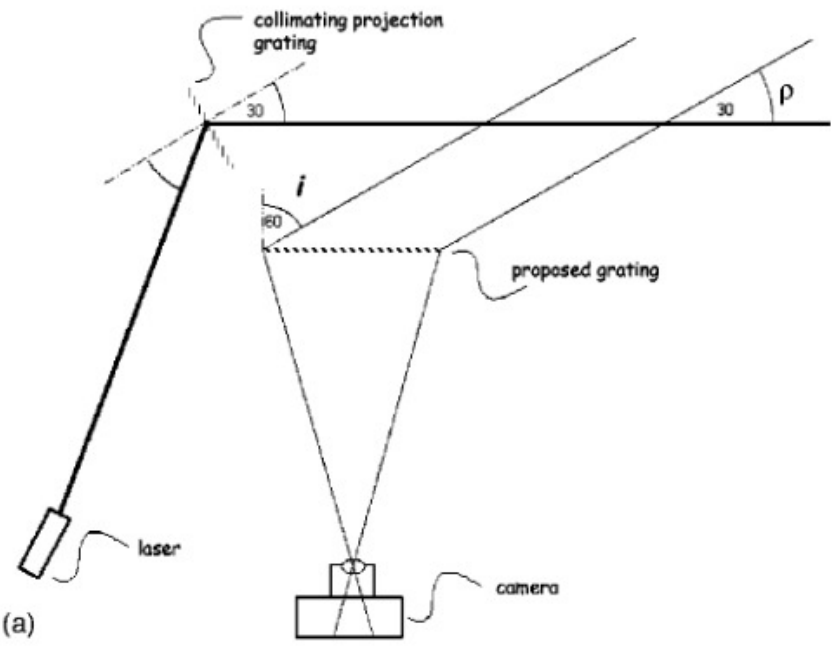

(a)

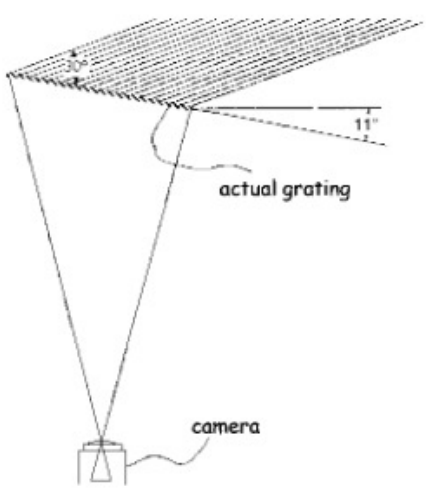

(b)

Fig. 15 (a) Post-Moly test bed. The diffraction images are formed without any keystone distortion. A holographic projection lens is used to form the collimated sheet of light projection. (b) Actual performance of the grating.



Fig. 16 Test block with a grating image made at a 40 deg occlusion liability superimposed inside the rectangle.

liability angle is actually $41 \mathrm{deg}$, because the grating was rotated an additional $11 \mathrm{deg}$, as per Fig. 15(b). By way of contrast, consider the triangulation range image in Fig. 17, made with the same test block at the same stand-off with the test block rotated to a 45-deg occlusion liability angle. The triangulation image shows perspective foreshortening even as it suffers a greater occlusion liability.

If an increase in occlusion liability can be tolerated, the method can be used to magnify beyond $1: 1$ by intersecting the structured light beam in an orientation perpendicular to the ray bundle. With diffraction angles approaching evanescence $(90 \mathrm{deg})$, such an approach promises to provide a means for 3-D microscopy. Such improvements may rest with the exploitation of grating materials other than silverhalide-depleted holographic plates. In this regard, we intend to investigate surface relief gratings, in part because they hold the promise of mass replication.

Unlike holography, our diffraction range finder must be integrated with appropriate software. Our research includes a software development component. In particular, the JAVA software that was pioneered in DiffCAD has been expanded to include scanner control, image processing, and 3 -D rendering applets.

The instrument in Fig. 18 was resident at the University of Bridgeport, where experimentation was conducted with a variety of localizer mechanisms other than Moly's mag-

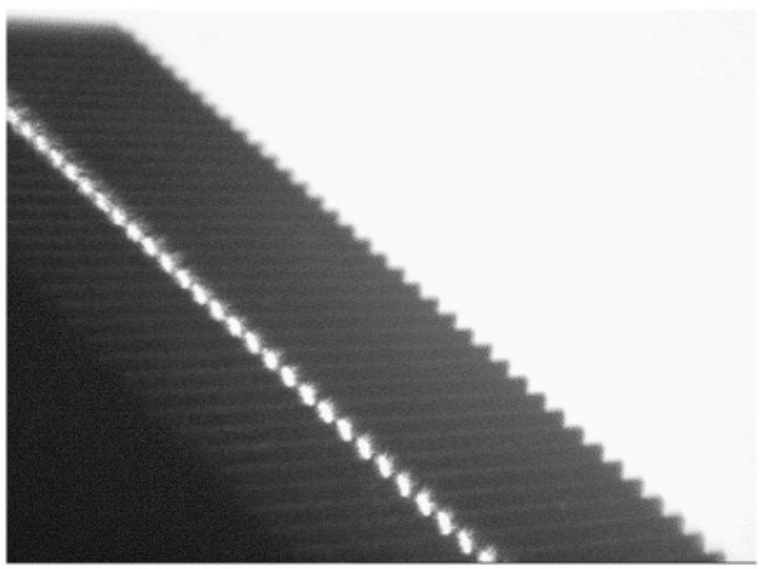

Fig. 17 Test block with triangulation image made at $45 \mathrm{deg}$ 


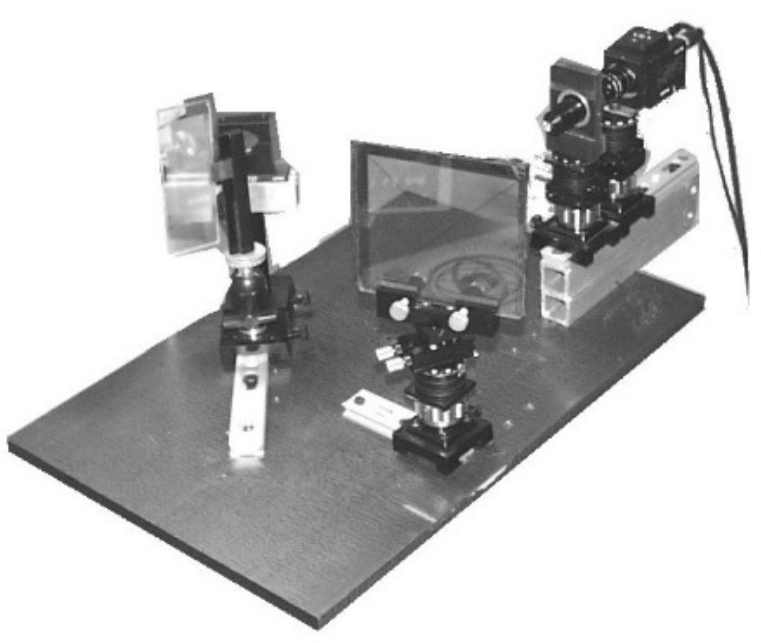

Fig. 18 Grating, camera, and laser projector assembly.

netic wave detectors. A rotary table and an $x-y$ table were used under embedded JAVA control to produce a series of scans that have been rendered in wireframe and texture mapped imagery illustrated by Fig. 19.

Other avenues of research and development are now being pursued. A license has been issued for the use of the diffraction range finder technology to Dimension Data ${ }^{14}$ of Novi, Michigan, aimed at the production of metrological instruments for use in the health and manufacturing sectors. We also have long-term interests in the graphic arts, and variations of Moly could find their way into the production of computer animation. Most of the contributors to our project also have professional credentials in the visual arts and music. This could not have been a coincidence.



Fig. 19 Chess piece scanned on rotary table rendered with marble texture in JAVA

\section{Acknowledgments}

Moly was built under Phase II National Science Foundation (NSF) SBIR Grant No. DMI-9420321 made to DeWitt Brothers Tool Company, Inc., in September 1995 through the NSF IRIS Directorate, and we wish to express our appreciation to DeWitt Brothers for their thorough and patient administration of our research project. The diffraction grating in Moly was made by Diffraction Ltd. of Waitsfield, Vermont. Follow-on gratings for the use of Dr. Lyon were made by Rudie Berkhout of New York City. An electronic interface, Surfa, for Moly's camera is manufactured by 3D Scanners of London, England, where special software for our project was written by Phil Hand with whom we share an interest in music and video art. The original design of Surfa was created and additional special software was written by Richard Monkhouse of Costronics Electronics, Hillingdon, Middlesex, England. The magnetic wave detector used in Moly is the Istotrak II, made by Polhemus, Inc. of Burlington, Vermont. We are appreciative of the guidance provided to us by the late Bill Polhemus who invented the magnetic wave technology. Special spatial coordinate software for the Isotrak II was written by David Holden of London, England. The molybdinum di-sulfide impregnated nylon shell of Moly, from which it obtained its name, was made by Paul Friedlander of London, England. Optical engineering was assisted by Donald W. Winrich of Northville, Michigan. Dimension Data of Novi, Michigan, has assisted us in meeting the cost of publishing our paper. Dr. Lyon's research is made possible, in part, by Instrumentation Improvement Grant No. DUE-9451520 from the National Science Foundation and by a Larsen Professor grant from the Larsen Fund. Thanks to Raul Mihali and to Dr. Lyon's other students who participated in the JAVA applet development project. We received marketing help from Randall Bradley of Hannacroix, New York, who was instrumental in our presentation at the National Aeronautics and Space Administration (NASA)-sponsored Technology 2007 competition in September 1997 at Photonics East where Moly was named SBIR Instrumentation and Sensor Technology of the Year.

\section{References}

1. T. DeWitt, "Pantomation demonstration," SIGGRAPH Video Film Rev. 2 (Section 9) (1982); T. DeWitt and P. Edelstein, "Pantomation a system for position tracking," in Proc. 2nd Symp. on Small Computers in the Arts, IEEE Computer Society, No. 455, pp. $61-70$ (1982); T. DeWitt, "Pantomation interface for the Apple II," in Proc. 3rd Symp. on Small Computers in the Arts, IEEE Computer Society, No. 499, pp. $25-29$ (1983)

2. T. D. DeWitt and D. A. Lyon, "A range finding method using diffraction gratings," Appl. Opt. 34, 2510-2521 (1995).

3. Unpublished videotape available from Tom Ditto, ditto@taconic.net Prior art in synchronous scanning methods is well exampled by $\mathrm{M}$. Rioux, "Laser range finder based on synchronized scanners," Appl. Opt. 23, 3837-3844 (1984).

4. S. Ray, The Lens in Action, Focal Press, p. 186 (1976)

5. T. Scheimpflug, "Method of distorting plane images by means of lenses or mirrors," U.S. Patent No. 751,847 (Feb. 9, 1904).

6. http://www.polhemus.com/hls1.htm; http://www.aranz.co.nz

7. E. N. Leith and J. Upatnieks, "Reconstructed wavefronts and communication theory," J. Opt. Soc. Am. 52, 1123-1130 (1962)

8. D. A. Lyon and H. V. Rao, JAVA Digital Signal Processing, M\&T Books (1998)

9. http://www.docjava.com

10. http://www praskovi.com.

11. http://mars-cam.com/frame/pinhole/v-zpl-xx.html.

12. http://www.3dscanners.com

13. http://rudieberkhout home mindspring.com.

14. http://www dimension-data.com. 


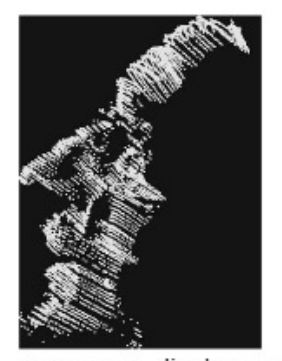

Tom Ditto is vice president of research with DeWitt Brothers Tool Company in New York. He has had an extensive career in the arts and his films and videos have been collected by major museums. Ditto is a fellow of the American Film Institute, the National Endowment for the Arts, and the Guggenheim Foundation. Ditto met Douglas A. Lyon in 1980 and worked with him for a decade at the R.P.I. Image Processing Laboratory. Ditto coauthored with Lyon numerous disclosures on diffraction range finding technology and they pioneered its continued development, culminating in the joint invention of the chirp grating technique used in the Moly prototype.

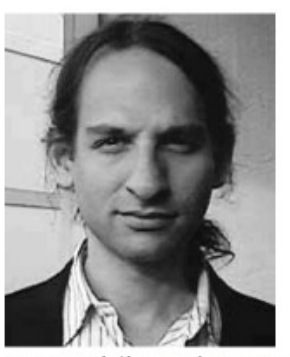

Douglas A. Lyon holds a $\mathrm{PhD}$ and is with the Computer Engineering Department at the University of Fairfield, Connecticut. He has been with the University of Bridgeport, the Jet Propulsion Laboratory's Artificial Intelligence R\&D Department, and AT\&T Bell Laboratories. Lyon met Tom Ditto in 1980 and worked with him for a decade at the R.P.I. Image Processing Laboratory. Lyon coauthored with Ditto numerous disclosures on diffraction range finding technology and they pioneered its continued development, culminating in the joint invention of the chirp grating technique used in the Moly prototype. 